\title{
Análise econômica e viabilidade energética da cultura do feijoeiro comum sob irrigação
}

\author{
Eder P. Gomes' ${ }^{1}$ Rodrigo A. Jordan' ${ }^{1}$, Anamari V. de A. Motomiya ${ }^{1}$, \\ Juliana B. Padua ${ }^{2}$, Guilherme A. Biscaro' ${ }^{1}$ \& Luciano O. Geisenhoff ${ }^{1}$
}

\begin{abstract}
RESUMO
Este trabalho foi realizado com o objetivo de avaliar a viabilidade econômica e energética da cultura do feijoeiro comum de terceira safra com lâminas de irrigação na região Noroeste do Paraná. O experimento foi implantado com seis lâminas de irrigação e quatro repetições, em delineamento de blocos ao acaso. A cultura do feijoeiro não foi viável economicamente sem irrigação. Os lucros cresceram com o aumento das lâminas de irrigação atingindo um lucro operacional total de $\mathrm{R} \$ 1.427,24 \mathrm{ha}^{-1}$ na lâmina de $333 \mathrm{~mm}$. O preço de equilíbrio sem irrigação foi de $R$ \$ 134,06 e R \$152,65 a saca, em curto e longo prazos, respectivamente. A irrigação favoreceu o balanço energético (energia extraída/energia utilizada) de maneira linear atingindo 1,63 na aplicação da maior lâmina $(333 \mathrm{~mm})$.
\end{abstract}

Palavras-chave: custo operacional de produção, manejo de irrigação, terceira safra, balanço de energia

\section{Economic analysis and energetic viability of the common bean crop under irrigation}

\begin{abstract}
This study was conducted to evaluate the economic and energetic viability of the third season of common bean under irrigation depths in the northwestern region of Parana. The experiment was established with six irrigation levels and four replications in a randomized block design. The bean crop was not economically viable without irrigation. Operating profit increased with increasing irrigation depths, reaching a total operating profit of $\mathrm{R} \$ 1,427.24 \mathrm{ha}^{-1}$ by $333 \mathrm{~mm}$ irrigation depth. The equilibrium price without irrigation was R\$134.06 and R\$152.65 bag $^{-1}$, short and long term, respectively. The irrigation favored the energy balance extracted (energy/used energy), reaching 1.63 in the application of greater depth (333 $\mathrm{mm})$.
\end{abstract}

Key words: operating cost of production, irrigation management, third season, energy balance 


\section{INTRODUÇÃO}

Na produção de feijão de terceira safra se destacam os estados de Minas Gerais, São Paulo e Goiás, predominando o feijão irrigado (CONAB, 2010). De maneira geral, a necessidade de água do feijoeiro com ciclo de 60 a 120 dias varia entre 300 a $500 \mathrm{~mm}$; no entanto, devido às condições de clima, época de semeadura e genótipos utilizados, em alguns casos a máxima produtividade pode ser atingida por meio de consumo hídrico menor que 250 mm (Jadoski et al., 2003; Arf et al., 2004).

Na maioria dos trabalhos o feijoeiro irrigado tem alcançado produtividades entre 1800 e $2500 \mathrm{~kg} \mathrm{ha}^{-1}$ (Silveira et al., 2001; Jadoski et al., 2003; Rapassi et al., 2003; Arf et al., 2004; Santana et al., 2009a).

O Paraná é o maior produtor brasileiro de feijão com $24 \%$ da produção nacional (CONAB, 2010); apesar de pouco destaque no cultivo de terceira safra, limitado em função da temperatura, normalmente feito sem irrigação. Mais de $70 \%$ da produção estão concentrados na região Sul do Estado e apenas 0,3\% na região Noroeste. A produção de terceira safra no Paraná representa apenas $1,5 \%$ da produção total paranaense, com produtividade da ordem de $727 \mathrm{~kg} \mathrm{ha}^{-1}$ (SEAB, 2010).

O zoneamento climático do Paraná demonstra que o noroeste pode ser utilizado para o feijão de terceira safra mas devido à baixa capacidade de armazenamento hídrico dos solos da região isto deve ser feito com uso do plantio direto e/ou irrigação (Ávila et al., 2010; Gomes et al., 2010)

Os trabalhos realizados com feijoeiro vêm demonstrando que a cultura é responsiva à irrigação, com ganhos expressivos de produtividade que compensam o aumento do custo de produção e viabilizam economicamente o uso da tecnologia (Silveira et al., 2001; Rapassi et al., 2003; Santana et al., 2009b).

Além da viabilidade econômica estudos de balanço energético em diferentes sistemas produtivos podem trazer subsídios para que a agropecuária brasileira se torne cada vez mais sustentável (Campos \& Campos, 2004). O balanço energético pode ser obtido pelo valor energético da produtividade sobre todos os gastos energéticos advindos da implantação da cultura (Albuquerque et al., 2007) constituindo importante instrumento de escolha tecnológica, evitando e substituindo os genótipos e sistemas produtivos com eficiência menor que um (Assenheimer et al., 2009). Na produção agrícola moderna, caracterizada por alto consumo de energia fóssil e recursos naturais (entrada), a produtividade (saída) normalmente precisa ser elevada para se ter um balanço de energia favorável (Hülsbergen et al., 2001).

A irrigação está entre as tecnologias que mais contribuem para o aumento da produtividade pois é responsável por $50 \%$ da produção agrícola mundial e ocupa apenas $18 \%$ da área colhida (Saturnino et al., 2010). No entanto a irrigação, que normalmente possibilita o aumento de produção e, por vezes, o aumento do lucro, incrementa a entrada (consumo) de energia no sistema de produção; por outro lado e dependendo do nível de produção, o balanço de energia poderá ser sustentável.

Em trabalho conduzido com a cultura do girassol, Jordan et al. (2012a) verificaram que o balanço de energia decresceu em função das lâminas de irrigação. Para a cultura da mamona,
Jordan et al. (2012b) verificaram que o balanço de energia não foi afetado pela irrigação; entretanto há, ainda, poucos estudos através dos quais se avalia o balanço energético da agricultura irrigada.

Este experimento foi desenvolvido com o objetivo de analisar indicadores econômicos e balanço energético da cultura do feijoeiro comum de terceira safra com lâminas de irrigação na região Noroeste do Paraná.

\section{Material e Métodos}

Este trabalho foi desenvolvido no ano agrícola de 2010 na área experimental da Universidade Estadual de Maringá, Campus do Arenito, em Cidade Gaúcha, PR, cujas coordenadas geográficas foram de $23^{\circ} 22^{\prime}$ '29" de latitude sul e $52^{\circ} 56^{\prime} 57^{\prime}$ ' de longitude oeste, a $404 \mathrm{~m}$ de altitude.

O clima da região, segundo classificação de Köppen, é do tipo Cfa (mesotérmico úmido, com chuvas abundantes no verão e inverno seco). Durante o período experimental a precipitação foi de $305,2 \mathrm{~mm}$ e $57 \%$ das chuvas (173,2 $\mathrm{mm}$ ) se concentraram nos vinte primeiros dias. A temperatura média foi de $24,6{ }^{\circ} \mathrm{C}$ e a umidade relativa média foi de 70,6\%. Na Figura 1 se encontram os valores de precipitação, temperatura e umidade relativa no decorrer do experimento.

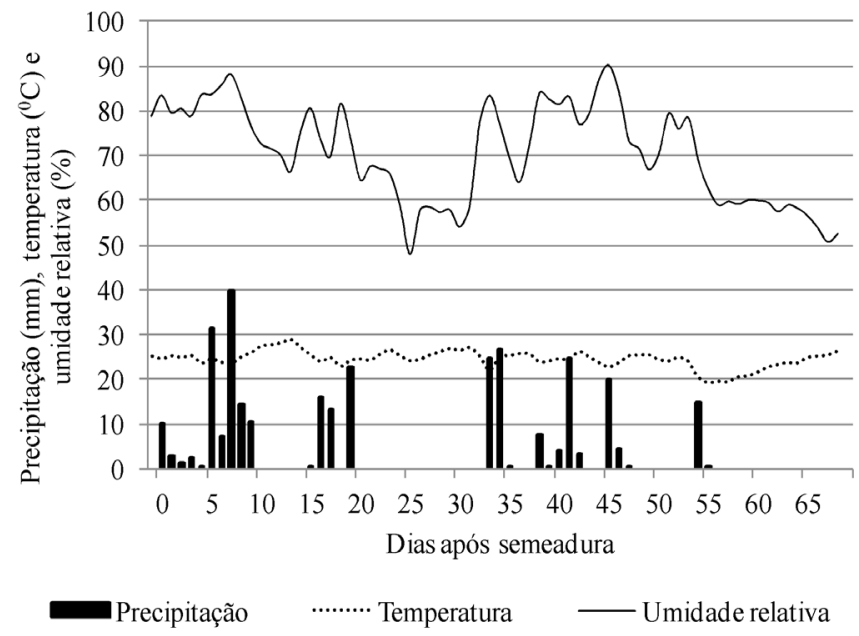

Figura 1. Valores de precipitação, temperatura e umidade relativa durante o período experimental

O solo da área experimental é classificado como Latossolo Vermelho Distrófico típico com horizonte A de textura arenosa e horizonte diagnóstico subsuperficial B latossólico de textura franco-arenosa (EMBRAPA, 2006). Os valores da análise química do solo referentes à profundidade de $0-0,20 \mathrm{~m}$ se encontram na Tabela 1.

No dia 09 de janeiro de 2010, com incorporação ao solo por meio de arado de disco reversível e grade niveladora, foram aplicadas $2 \mathrm{t} \mathrm{ha}^{-1}$ de calcário dolomítico com PRNT de $80 \%$. Na adubação de semeadura aplicaram-se $200 \mathrm{~kg} \mathrm{ha}^{-1}$ do formulado 0-20-20 e na adubação de cobertura, $110 \mathrm{~kg} \mathrm{ha}^{-1} \mathrm{de}$ nitrogênio na forma de ureia, parcelada em três vezes, aos 15, 25 e 35 dias após semeadura.

A cultura foi semeada no dia 9 de fevereiro de 2010 a cinco $\mathrm{cm}$ de profundidade, com linhas espaçadas $0,5 \mathrm{~m}$ e densidade 
Tabela 1. Análise química do solo da área experimental na camada de $0-0,20 \mathrm{~m}$

\begin{tabular}{ccccccc}
\hline $\mathbf{p H}$ & $\mathbf{P}$ & $\mathbf{H}^{+}+\mathbf{A l}^{3+}$ & $\mathbf{A l}^{3+}$ & $\mathbf{C a}^{2+}$ & $\mathbf{M g}^{2+}$ & $\mathbf{K}^{+}$ \\
\cline { 3 - 7 } & $\mathbf{\mathbf { H } _ { 2 } \mathbf { O } )}$ & $\mathbf{m g ~ d m}$ & \multicolumn{5}{c}{$\mathbf{c m o l}_{\mathbf{c}} \mathbf{~ d m}^{-3}$} \\
6,4 & 20,20 & 2,73 & 0,00 & 1,18 & 0,58 & 0,13 \\
\hline
\end{tabular}

de 16 sementes por metro. Utilizou-se a cultivar IPR Colibri "carioca", grupo de maturação precoce e tipo I.

O trabalho foi implantado com seis tratamentos (lâminas de irrigação) e quatro repetições, com delineamento de blocos ao acaso. A linha de irrigação em PVC de $50 \mathrm{~mm}$ foi montada paralela às linhas de semeadura, contendo cinco aspersores espaçados $6 \mathrm{~m}$ (coincidente ao comprimento da parcela). Cada parcela foi constituída por cinco linhas de plantas totalizando uma área de $15 \mathrm{~m}^{2}$ (6 x 2,5 m).

Perpendicular à linha de irrigação e no centro de cada parcela irrigada foi colocado um coletor de $10 \mathrm{~cm}$ de diâmetro a um metro do solo. Outros coletores foram instalados a 2, 4, 6,8 e 10 m, representando as lâminas aplicadas: L5, L4, L3, L2 e L1, respectivamente. Além do alcance do aspersor foram instaladas as parcelas testemunha (L0). Os coletores dispostos a $6 \mathrm{~m}$ (L3) foram estabelecidos como referência para o manejo de irrigação. Anterior ao período experimental, com os aspersores em funcionamento, verificou-se o volume desses coletores durante uma hora obtendo-se a intensidade de aplicação (IA) de $12 \mathrm{~mm} \mathrm{~h}^{-1}$.

A partir da umidade do solo as irrigações foram realizadas duas vezes por semana (segundas e quintas-feiras) a partir das $16 \mathrm{~h}$ para atenuar a influência do vento, exceto na presença de chuva ou com umidade do solo superior à capacidade de campo. O tempo de irrigação foi obtido pela razão entre a lâmina de referência (L3) e intensidade de aplicação (IA).

A lâmina de irrigação de referência (L3) foi determinada a partir da diferença entre a umidade volumétrica na capacidade de campo $\left(\theta_{\text {cc }}\right)$ e atual $\left(\theta_{\mathrm{a}}\right)$, multiplicada pela profundidade efetiva da raiz (Z), considerada igual a $200 \mathrm{~mm}$ nos primeiros 15 dias e depois igual a $400 \mathrm{~mm}$ até o fim do experimento. Após cada irrigação as lâminas aplicadas foram medidas nos coletores de todos os tratamentos (Tabela 2).

A umidade na capacidade de campo $\left(\theta_{\text {cc }}\right)$, igual $0,215 \mathrm{~cm}^{3}$ $\mathrm{cm}^{-3}$ para a camada de 0 a a $40 \mathrm{~cm}$, foi obtida por meio de

Tabela 2. Umidade volumétrica atual $(\theta a)$ no tratamento L3 e lâminas de irrigação (L5, L4, L3, L2 e L1) aplicadas na cultura do feijoeiro

\begin{tabular}{ccccccrr}
\hline \multirow{2}{*}{ Dia } & \multirow{2}{*}{$\mathbf{D A S}$} & $\begin{array}{c}\theta \mathbf{a} \\
\mathbf{( c m}^{\mathbf{3}} \mathbf{c m}^{-3} \mathbf{)}\end{array}$ & $\mathbf{L 5}$ & $\mathbf{L 4}$ & $\mathbf{L 3}$ & $\mathbf{L 2}$ & $\mathbf{L 1}$ \\
\hline $22 / 02$ & 13 & 0,1967 & 13,2 & 10,3 & $\mathbf{( m \mathbf { m } )}$ & & \\
$01 / 03$ & 20 & 0,1718 & 28,0 & 20,2 & 17,3 & 6,2 & 5,1 \\
$05 / 03$ & 24 & 0,1664 & 34,6 & 22,0 & 19,4 & 16,9 & 13,5 \\
$08 / 03$ & 27 & 0,1827 & 20,3 & 15,3 & 12,9 & 11,6 & 6,8 \\
$11 / 03$ & 30 & 0,1656 & 35,2 & 22,3 & 19,7 & 15,8 & 10,3 \\
$18 / 03$ & 37 & 0,1719 & 27,7 & 20,0 & 17,2 & 17,7 & 13,3 \\
$25 / 03$ & 44 & 0,1704 & 32,3 & 22,5 & 17,8 & 15,4 & 9,9 \\
$29 / 03$ & 48 & 0,1500 & 46,5 & 31,8 & 26,1 & 21,3 & 2,2 \\
$01 / 04$ & 51 & 0,1719 & 27,7 & 20,0 & 17,2 & 17,7 & 13,3 \\
$08 / 04$ & 58 & 0,1704 & 32,3 & 22,5 & 17,8 & 15,4 & 9,9 \\
$12 / 04$ & 62 & 0,1656 & 35,2 & 22,3 & 19,7 & 15,8 & 10,3 \\
\hline Total & & & 333,0 & 229,2 & 192,4 & 171,8 & 104,1 \\
\hline DAS - dias após semeadura & & & & &
\end{tabular}

DAS - dias após semeadura curva de retenção ajustada pelo modelo de Genutchen (1980). Adotou-se tensão de água correspondente a solo arenoso, ou seja, igual a $10 \mathrm{kPa}$, conforme Albuquerque (2010).

Nos dias de irrigação amostras de solo foram retiradas para determinação da umidade volumétrica atual $\left(\theta_{\mathrm{a}}\right)$ utilizando-se trado de $25 \mathrm{~mm}$ de diâmetro e encaminhadas ao laboratório onde foram pesadas em balança analítica com resolução de $0,001 \mathrm{~g}$ e posteriormente levadas ao forno de micro-ondas, onde permaneceram por 30 min sob potência da ordem de $1300 \mathrm{~W}$; em seguida, as amostras de solo foram retiradas do forno de micro-ondas e novamente pesadas obtendo-se, por diferença, a massa de água que, dividida pela massa seca do solo, originou a umidade gravimétrica atual $\left(\mathrm{U}_{\mathrm{a}}\right)$.

A umidade volumétrica atual $\left(\theta_{\mathrm{a}}\right)$ foi obtida por meio do produto da umidade gravimétrica atual $\left(\mathrm{U}_{\mathrm{a}}\right)$ e densidade do solo (Ds), sendo esta determinada por meio de amostras retiradas com o uso de cilindros volumétricos e posteriormente secadas em estufa a $105{ }^{\circ} \mathrm{C}$ durante $30 \mathrm{~h}$ obtendo-se o valor médio na camada de 0-40 $\mathrm{cm}$ da ordem de $1,5 \mathrm{~g} \mathrm{~cm}^{-3}$. Seis amostras foram coletadas na diagonal da área experimental por meio de cilindros volumétricos, três na camada de $0-20 \mathrm{~cm}$ e três na camada de $20-40 \mathrm{~cm}$

A colheita do feijão foi realizada no dia 20 de abril estimando-se a produtividade com correção de umidade de $13 \%$. A produtividade foi submetida às análises de variância e regressão, a $5 \%$ de probabilidade, utilizando-se o software Assistat 7.6 beta (Silva \& Azevedo, 2010).

A análise econômica foi feita a partir do custo operacional de produção total (COT) e o custo operacional efetivo (COE) utilizando-se de cotações do mercado. Na composição do COE foram considerados os gastos com insumo, operações agrícolas, mão-de-obra, impostos e despesas administrativas. Obteve-se o COT pela soma do COE mais depreciação do capital (Martin et al., 1994).

$$
\mathrm{COT}=\mathrm{COE}+\mathrm{DC}
$$

em que:

$$
\begin{aligned}
& \text { COT - custo operacional total, } \mathrm{R} \$ \mathrm{ha}^{-1} \\
& \mathrm{COE} \text { - custo operacional efetivo, } \mathrm{R} \$ \mathrm{ha}^{-1} \\
& \text { DC - depreciação do capital, } \mathrm{R} \$ \mathrm{ha}^{-1}
\end{aligned}
$$

Ao custo operacional efetivo dos tratamentos irrigados $\left(\mathrm{COE}_{\mathrm{i}}\right)$ acrescentaram-se os gastos com energia e manutenção do sistema de irrigação decorrido durante o ciclo da cultura. Por se tratar de um sistema autopropelido (simulação com pivô central) não se considerou acréscimo de mão-de-obra (o hífen continua) em função da irrigação; na região ainda não se cobra pelo uso da água.

$$
\mathrm{COE}_{\mathrm{i}}=\mathrm{COE}+\mathrm{CE}+\mathrm{CM}
$$

em que:

$\mathrm{COE}_{\mathrm{i}}$ - custo operacional efetivo sob irrigação, $\mathrm{R} \$ \mathrm{ha}^{-1}$

$\mathrm{CE}$ - custo da energia elétrica por ciclo de produção, $\mathrm{R} \$ \mathrm{ha}^{-1}$

$\mathrm{CM}$ - custo de manutenção por ciclo de produção, $\mathrm{R} \$ \mathrm{ha}^{-1}$

A partir das lâminas aplicadas a simulação foi baseada na potência, rendimento mecânico e fator de potência do motor 
elétrico de uma bomba d'água de um pivô central para 100 ha, com potência elétrica requerida para bombeamento de aproximadamente $1,472 \mathrm{~kW} \mathrm{ha}^{-1}$, valor este considerado no cálculo do custo de energia. Consideraram-se, ainda, uma intensidade de aplicação de $0,33 \mathrm{~mm} \mathrm{~h}^{-1}$ e custo de manutenção (CM) para pivô-central estimado a $1,5 \%$ ao ano (Frizzone et al., 2005). A energia elétrica foi taxada conforme a tarifa rural estabelecida pela Companhia Paranaense de Energia, igual R\$ $0,16 \mathrm{~kW} \mathrm{~h}^{-1}$ (COPEL, 2010).

$\mathrm{O}$ custo de energia (CE) foi estimado da seguinte forma:

$$
\mathrm{CE}=1,472 \times \mathrm{PE} \times \mathrm{H}
$$

em que:

$$
\begin{array}{ll}
\mathrm{PE} & \text { - preço da energia, } \mathrm{R} \$ \mathrm{kWh}^{-1} \\
\mathrm{H} & \text { - tempo de irrigação por ciclo de produção, } \mathrm{h}
\end{array}
$$

O lucro operacional efetivo (LOE), que representa a viabilidade econômica em curto prazo, foi obtido pela diferença entre a receita bruta (RB) e o custo operacional efetivo (COE):

$$
\mathrm{LOE}=\mathrm{RB}-\mathrm{COE}
$$

em que:

LOE - lucro operacional efetivo, $\mathrm{R} \$ \mathrm{ha}^{-1}$

$\mathrm{RB}$ - receita bruta, $\mathrm{R} \$ \mathrm{ha}^{-1}$

O lucro operacional total (LOT), que representa a viabilidade econômica em longo prazo, foi obtido pela diferença entre a receita bruta (RB) e o custo operacional total (COT):

$$
\mathrm{LOT}=\mathrm{RB}-\mathrm{COT}
$$

em que:

$$
\text { LOT - lucro operacional total, } \mathrm{R} \$ \mathrm{ha}^{-1}
$$

No caso dos tratamentos irrigados calculou-se, adicionalmente, a depreciação do capital (DC) pelo método do fator de recuperação do capital (Marques \& Coelho, 2003), desconsiderando o valor residual. Adotou-se vida útil de 20 anos (n) e capacidade de uso igual a $2000 \mathrm{~h} \mathrm{ano}^{-1}$. Considerouse o sistema de irrigação do tipo Pivô-Central (por ser o mais utilizado em irrigação de feijão) admitindo-se um valor de R\$ $4500,00 \mathrm{ha}^{-1}$, conforme média de preço praticado no ano de 2010 e se empregando taxa de juros (J) de 6,5\% ao ano, tomada com base na linha de financiamento para máquinas e equipamentos agrícolas (FINAME, 2010).

$$
\mathrm{DC}=\left[\frac{\mathrm{C} \times \mathrm{J}(\mathrm{J}+1)^{\mathrm{n}}}{(1+\mathrm{J})^{\mathrm{n}}-1}\right] \times \mathrm{F}
$$

em que:

$$
\begin{array}{ll}
\text { DC } & \text { - depreciação de capital, } R \$ h^{-1} \\
\text { C } & \text { - custo de aquisição do capital, } R \$ h^{-1} \\
\text { J } & \text { - taxa anual de juros, decimal } \\
\text { n } & \text { - vida útil, anos } \\
\text { F } & \text { - relação entre horas de uso por ciclo e horas por ano, }
\end{array}
$$
decimal
Além da análise econômica realizada por meio do custo operacional, foram propostos, no intuito de ampliar a discussão, quatro indicadores econômicos:

Relação benefício custo $(\mathrm{B} / \mathrm{C})$ : relação entre a receita e o custo de produção, representando prejuízo quando o valor é menor que 1.

$$
\mathrm{B} / \mathrm{C}=\frac{\mathrm{RB}}{\mathrm{CO}}
$$

em que:

B/C - relação benéficio custo, adimensional

$\mathrm{CO}$ - custo operacional de produção, efetivo ou total, $\mathrm{R} \$ \mathrm{ha}^{-1}$

Ponto de nivelamento (PN): definida como produtividade mínima necessária para cobrir o custo operacional de produção.

$$
\mathrm{PN}=\frac{\mathrm{CO}}{\mathrm{PU}}
$$

em que:

PN - ponto de nivelamento, $\mathrm{sc} \mathrm{ha}^{-1}$

$\mathrm{PU}$ - preço unitário, $\mathrm{R} \$ \mathrm{sc}^{-1}$

Preço de equilíbrio (PE): definido pelo preço mínimo necessário para pagar o custo operacional de produção.

$$
\mathrm{PE}=\frac{\mathrm{CO}}{\mathrm{PROD}}
$$

em que:

$\mathrm{PE}$ - preço de equilíbrio, $\mathrm{R} \$ \mathrm{sc}^{-1}$

PROD - produtividade, $\mathrm{sc} \mathrm{ha}^{-1}$

Índice de lucratividade (IL): estabelece a relação percentual entre lucro e receita.

$$
\mathrm{IL}=\frac{\mathrm{LO}}{\mathrm{RB}} \times 100
$$

em que:

IL - índice de lucratividade, \%

LO - lucro operacional efetivo ou total, $\mathrm{R} \$ \mathrm{ha}^{-1}$

A análise da viabilidade energética foi realizada por meio de balanço de energia utilizando-se da metodologia de análise de processos (Hülsbergen et al., 2001):

$$
\mathrm{BE}=\frac{\mathrm{EE}}{\mathrm{EU}}
$$

em que:

BE - balanço de energia, adimensional

EE - energia extraída, $\mathrm{MJ} \mathrm{ha}^{-1}$

EU - energia utilizada, $\mathrm{MJ} \mathrm{ha}^{-1}$

A EE foi estimada com base na produtividade do feijoeiro considerando-se equivalência energética das sementes igual a $16,8 \mathrm{MJ} \mathrm{kg}^{-1}$ (Rios et al., 2003). 
A EU foi estimada da seguinte forma:

$$
\mathrm{EU}=\mathrm{DE}+\mathrm{EI}+\mathrm{EMO}+\mathrm{EEL}
$$

em que:

DE - depreciação energética dos equipamentos, $\mathrm{MJ} \mathrm{ha}^{-1}$

EI - energia demanda pelo uso de insumos, $\mathrm{MJ} \mathrm{ha}^{-1}$

EMO - energia empregada na mão-de-obra, $\mathrm{MJ} \mathrm{ha}^{-1}$

EEL - energia consumida na forma de eletricidade, $\mathrm{MJ} \mathrm{ha}^{-1}$

A energia demandada pelo uso de insumos foi adotada conforme valores recomendados por Hülsbergen et al. (2001) e Melo et al. (2007).

No caso dos equipamentos não propelidos (implementos) a DE foi calculada da seguinte maneira:

$$
\mathrm{DE}_{\mathrm{ENP}}=\frac{57,2 \mathrm{M}}{\mathrm{n}} \mathrm{F}
$$

Já no caso dos equipamentos propelidos (trator e pivôcentral) a DE foi calculada da seguinte forma:

$$
\mathrm{DE}_{\mathrm{EP}}=\frac{69,8 \mathrm{M}}{\mathrm{n}} \mathrm{F}
$$

em que:

$\mathrm{DE}_{\mathrm{ENP}}$ - depreciação energética dos equipamentos não propelidos, $\mathrm{MJ} \mathrm{ha}^{-1}$ $\mathrm{MJ} \mathrm{ha}^{-1}$

$\mathrm{DE}_{\mathrm{EP}}$ - depreciação energética dos equipamentos propelidos,

M - massa das máquinas e equipamentos, $\mathrm{kg}$

n - vida útil, h

A massa das máquinas e os equipamentos foram adotados conforme recomendação de Assenheimer et al. (2009) e a vida útil de acordo com Checheto et al. (2010). A massa do sistema de irrigação do tipo pivô central, igual a $57,2 \mathrm{~kg} \mathrm{~m}^{-1}$, conforme Empresa Valmont Indústria e Comércio Ltda.

\section{Resultados e Discussão}

Na Tabela 3 estão listados os principais componentes do custo de produção. Os gastos com insumos representaram $51,94 \%$ do custo operacional efetivo (COE) e $45,61 \%$ do custo operacional total (COT). Johann et al. (2010) também observaram que o maior percentual do custo operacional recai sobre os insumos.

\begin{tabular}{|c|c|}
\hline Componentes do custo & $\begin{array}{c}\text { Valor } \\
\left(\mathrm{R} \$ \mathrm{ha}^{-1}\right)\end{array}$ \\
\hline Insumos $(A)$ & 964,51 \\
\hline Operações agrícolas (B) & 319,46 \\
\hline Mão-de-obra (C) & 391,63 \\
\hline Impostos e despesas administrativas (D) & 181,42 \\
\hline Depreciação de capital das máquinas e implementos (E) & 257,52 \\
\hline Custo operacional efetivo (COE) $=A+B+C+D$ & 1857,02 \\
\hline Custo operacional total $(\mathrm{COT})=\mathrm{A}+\mathrm{B}+\mathrm{C}+\mathrm{D}+\mathrm{E}$ & 2114,54 \\
\hline
\end{tabular}

Tabela 3. Custo de produção da cultura do feijoeiro sem irrigação

A produtividade respondeu de maneira linear à lâmina de irrigação (PROD = 4,72 LI + 838,15; $\left.\mathrm{R}^{2}=0,9236 ; \mathrm{P}<0,01\right)$ atingindo $2401,78 \mathrm{~kg} \mathrm{ha}^{-1}$ (Tabela 4). A produtividade está corroborada por diversos trabalhos de feijoeiro irrigado que obtiveram rendimentos entre 1800 e $2500 \mathrm{~kg} \mathrm{ha}^{-1}$ (Silveira et al., 2001; Jadoski et al., 2003; Rapassi et al., 2003; Arf et al., 2004; Santana et al. 2009a).

Sem irrigação a cultura do feijoeiro obteve prejuízos iguais a $\mathrm{R} \$ 471,77$ e $\mathrm{R} \$ 729,29$, respectivamente para LOE e LOT (Tabela 4), inviabilizando a atividade em curto e longo prazos.

Os lucros cresceram à medida que se aumentaram as lâminas de irrigação e o LOT obtido por hectare pela aplicação da maior lâmina $(333 \mathrm{~mm})$, igual $\mathrm{R} \$ 1410,35$ se assemelha ao valor encontrado por Santana et al. (2009b) que obtiveram, em experimento de feijoeiro sob irrigação, um LOT de R $\$ 1304,89$ ha $^{-1}$. A aplicação da menor lâmina $(104,1 \mathrm{~mm})$ acarretou prejuízo em longo prazo (Tabela 4).

Os índices de lucratividade (IL) no cultivo do feijoeiro sem irrigação foram negativos em curto e longo prazos (Tabelas $5 \mathrm{e}$

\begin{tabular}{|c|c|c|c|c|c|c|c|}
\hline Tratamentos & (m) & 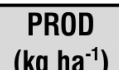 & RB & $\mathrm{COE}$ & $\frac{\text { COT }}{\left(\mathrm{C}^{-1} \mathrm{ha}^{-1}\right)}$ & LOE & LOT \\
\hline L0 & 0 & 831,15 & 1385,00 & 1857,02 & 2114,54 & $-471,77$ & $-729,29$ \\
\hline L1 & 104,1 & 1322,15 & 2203,58 & 1941,96 & 2263,99 & 261,62 & $-60,41$ \\
\hline L2 & 171,8 & 1641,46 & 2735,77 & 1997,20 & 2361,19 & 738,57 & 374,58 \\
\hline L3 & 192,4 & 1738,62 & 2897,71 & 2014,01 & 2390,76 & 883,69 & 506,94 \\
\hline L4 & 229,2 & 1912,19 & 3186,99 & 2044,04 & 2443,60 & 1142,95 & 743,40 \\
\hline L5 & 333,0 & 2401,78 & 4002,96 & 2128,74 & 2592,62 & 1874,23 & 1410,35 \\
\hline
\end{tabular}
6) iguais a -34,06 e -52,65\%, respectivamente. Na aplicação da

Tabela 4. Custo e lucro operacional do feijoeiro sob lâminas de irrigação

\begin{tabular}{|c|c|c|c|c|c|c|c|}
\hline Tratamentos & $\mathrm{LI}(\mathrm{mm})$ & PROD $\left(\mathrm{kg} \mathrm{ha}^{-1}\right)$ & PROD (sc ha-1) & $B / C$ & PN $\left(s c h a^{-1}\right)$ & PE $\left(R \$ h a^{-1}\right)$ & $\overline{I L(\%)}$ \\
\hline LO & 0 & 831,15 & 13,85 & 0,75 & 18,57 & 134,06 & $-34,06$ \\
\hline L1 & 104,1 & 1322,15 & 22,04 & 1,13 & 19,42 & 88,13 & 11,87 \\
\hline L2 & 171,8 & 1641,46 & 27,36 & 1,37 & 19,97 & 73,00 & 27,00 \\
\hline L3 & 192,4 & 1738,62 & 28,98 & 1,44 & 20,14 & 69,50 & 30,50 \\
\hline L4 & 229,2 & 1912,19 & 31,87 & 1,56 & 20,44 & 64,14 & 35,86 \\
\hline L5 & 333,0 & 2401,78 & 40,03 & 1,88 & 21,29 & 53,18 & 46,82 \\
\hline
\end{tabular}

LI - lâmina de irrigação; PROD - produtividade; RB - receita bruta; COE - custo operacional efetivo; COT - custo operacional total; LOE - lucro operacional efetivo; LOT - lucro operacional total

Tabela 5. Indicadores econômicos do feijoeiro sob irrigação para viabilidade em curto prazo

LI - lâmina de irrigação; PROD - produtividade; B/C - relação benefício custo; PN - ponto de nivelamento; PE - preço de equilíbrio; IL - índice de lucratividade 
Tabela 6. Indicadores econômicos do feijoeiro sob irrigação para viabilidade em longo prazo

\begin{tabular}{|c|c|c|c|c|c|c|c|}
\hline Tratamentos & LI (mm) & PROD $\left(\mathrm{kg} \mathrm{ha}^{-1}\right)$ & PROD (sc ha-1) & $B / C$ & PN (sc ha-1) & PE $\left(R \$ h^{-1}\right)$ & IL (\%) \\
\hline LO & 0 & 831,15 & 13,85 & 0,66 & 21,15 & 152,65 & $-52,65$ \\
\hline L1 & 104,1 & 1322,15 & 22,04 & 0,97 & 22,64 & 102,74 & $-2,74$ \\
\hline L2 & 171,8 & 1641,46 & 27,36 & 1,16 & 23,61 & 86,31 & 13,69 \\
\hline L3 & 192,4 & 1738,62 & 28,98 & 1,21 & 23,91 & 82,51 & 17,49 \\
\hline L4 & 229,2 & 1912,19 & 31,87 & 1,30 & 24,44 & 76,67 & 23,33 \\
\hline L5 & 333,0 & 2401,78 & 40,03 & 1,54 & 25,93 & 64,77 & 35,23 \\
\hline
\end{tabular}

LI - lâmina de irrigação; PROD - produtividade; B/C - relação benefício custo; PN - ponto de nivelamento; PE - preço de equilíbrio; IL - índice de lucratividade

maior lâmina de irrigação obteve-se um IL de 35,23\% em longo prazo. Este resultado está de acordo com valores encontrados por Rapassi et al. (2003) que obtiveram, para o feijoeiro sob irrigação IL, entre 30 e $40 \%$ em longo prazo.

A relação $\mathrm{B} / \mathrm{C}$ foi proporcional à lâmina de irrigação atingindo 1,88 e 1,54 em curto e longo prazos, respectivamente (Tabelas 5 e 6). Segundo Silveira et al. (2001) a irrigação em feijoeiro tem promovido relações $\mathrm{B} / \mathrm{C}$ em curto prazo entre 1,67 e 1,97, o que corrobora com o resultado deste trabalho.

O preço de equilíbrio (PE) sem irrigação foi de R $\$ 134,06$ e R $\$ 152,65$ a saca em curto e longo prazos, respectivamente valores esses superiores ao preço adotado por saca neste trabalho, igual $\mathrm{R} \$ 100,00$, ratificando a inviabilidade do cultivo do feijoeiro sem irrigação na terceira safra.

A produtividade obtida sem irrigação, da ordem de 13,85 sc $\mathrm{ha}^{-1}$, é condizente com o rendimento de terceira safra de feijoeiro do Estado do Paraná (SEAB 2010); no entanto, abaixo do ponto de nivelamento $(\mathrm{PN})$, que define a produtividade mínima necessária para cobrir o custo de produção, igual 18,57 e 21,15 sc ha-1 ${ }^{-1}$ em curto e longo prazos, respectivamente.

A energia utilizada (EU) para produção da cultura do feijoeiro sem irrigação foi de 13791,3 $\mathrm{MJ} \mathrm{ha}^{-1}$ (Tabela 10) sendo 290,7 MJ consumidos na forma de depreciação energética (Tabela 7) e 13390 na forma de energia demandada por insumos (Tabela 8), responsável por $97,9 \%$ da EU.

Tabela 7. Depreciação energética (DE) em função do tempo de uso de máquinas e equipamentos utilizados no cultivo do feijoeiro em sistema convencional

\begin{tabular}{lcccc}
\hline $\begin{array}{c}\text { Máquinas e } \\
\text { implementos }\end{array}$ & $\begin{array}{c}\text { Massa } \\
\mathbf{( k g )}\end{array}$ & $\begin{array}{c}\text { Vida útil } \\
\text { (h) }\end{array}$ & $\begin{array}{c}\text { Tempo de } \\
\text { uso (h) }\end{array}$ & $\begin{array}{c}\text { DE } \\
\text { (MJ ha-1) }\end{array}$ \\
Trator $80 \mathrm{cV}$ & 3000 & 12000 & 5,3 & 92,5 \\
Grade & 1000 & 3000 & 0,6 & 11,4 \\
Semeadora & 2000 & 1500 & 0,8 & 61,0 \\
Adubadora & 1000 & 1500 & 0,6 & 22,9 \\
Pulverizador & 1000 & 1500 & 1,3 & 49,6 \\
Recolhedora & 2000 & 3000 & 0,8 & 30,5 \\
Arado & 1000 & 3000 & 0,6 & 11,4 \\
Espalhador de calcário & 1000 & 3000 & 0,6 & 11,4 \\
Total & & & & 290,7 \\
\hline
\end{tabular}

Trabalhos conduzidos por Checheto et al. (2010) e Jordan et al. (2012a) também demonstraram que os insumos são os principais responsáveis pela entrada de energia (energia utilizada) na produção agrícola pois, no primeiro caso, em experimento conduzido com a cultura da mamona a energia demandada por insumos (EI) representou $97,4 \%$ da energia utilizada (EU) e, no segundo caso, em experimento conduzido com a cultura do girassol, os autores observaram que a energia demandada por insumos (EI), na ausência de irrigação foi da ordem de $90 \%$ da energia utilizada (EU).
Tabela 8. Energia demandada pelo uso de insumos (EI) utilizados no cultivo do feijoeiro

\begin{tabular}{lcccc}
\hline \multicolumn{1}{c}{ Insumos } & Unidade & $\begin{array}{c}\text { Energia } \\
\text { unitária (MJ) }\end{array}$ & $\begin{array}{c}\text { Quantidade } \\
\left.\text { (unidade ha }{ }^{-1}\right)\end{array}$ & $\begin{array}{c}\text { El } \\
\left(\mathbf{M J ~ h a}^{-1}\right)\end{array}$ \\
Nitrogênio $(\mathrm{N})$ & $\mathrm{kg}$ & 50,3 & 50 & 2515 \\
Fósforo $\left(\mathrm{P}_{2} \mathrm{O}_{5}\right)$ & $\mathrm{kg}$ & 12,6 & 40 & 504 \\
Potássio $\left(\mathrm{K}_{2} \mathrm{O}\right)$ & $\mathrm{kg}$ & 6,8 & 40 & 272 \\
Calcário & $\mathrm{kg}$ & 1,2 & 2000 & 2400 \\
Herbicidas & $\mathrm{L}$ & 400 & 2,7 & 1080 \\
Inseticidas & $\mathrm{L}$ & 400 & 2,2 & 880 \\
Fungicidas & $\mathrm{L}$ & 400 & 5,1 & 2040 \\
Sementes & $\mathrm{kg}$ & 16,8 & 60 & 1008 \\
Óleo diesel & $\mathrm{L}$ & 35,5 & 75,8 & 2691 \\
Total & & & & 13390 \\
\hline
\end{tabular}

Mesmo nos tratamentos irrigados e apesar do comportamento decrescente, os insumos continuaram tendo a maior participação na energia utilizada diminuindo para $54 \%$ na aplicação maior lâmina (Tabela 8). Este comportamento difere de Jordan et al. (2012a), em experimento com girassol, em que nos tratamentos irrigados a energia elétrica (EEL) e a depreciação energética (DE) obtiveram maior participação na energia utilizada (EU). $\mathrm{Na}$ Tabela 9 se encontram os valores de EEL e DE em função das lâminas de irrigação.

Tabela 9. Energia consumida em forma de eletricidade (EEL) e depreciação energética (DE) do feijoeiro sob lâminas de irrigação (LI)

\begin{tabular}{ccccc}
\hline $\begin{array}{c}\text { LI } \\
(\mathbf{m m})\end{array}$ & $\begin{array}{c}\text { EEL } \\
\left.\mathbf{( k W h ~ h a - 1}^{-1}\right)\end{array}$ & $\begin{array}{c}\text { EEL } \\
\mathbf{( M J ~ h a}^{-1} \mathbf{)}\end{array}$ & $\begin{array}{c}\text { Tempo de } \\
\mathbf{u s o} \mathbf{( h )}\end{array}$ & $\begin{array}{c}\text { DE } \\
\mathbf{( M J ~ h a}^{-1} \mathbf{)}\end{array}$ \\
104,1 & 464,35 & 1671,7 & 315,45 & 1775,5 \\
171,8 & 766,33 & 2758,8 & 520,61 & 2930,2 \\
192,4 & 858,22 & 3089,6 & 583,03 & 3281,6 \\
229,2 & 1022,37 & 3680,5 & 694,55 & 3909,2 \\
333,0 & 1485,38 & 5347,4 & 1009,10 & 5679,7 \\
\hline
\end{tabular}

$\mathrm{O}$ valor de EU para o tratamento sem irrigação $(\mathrm{LI}=0)$, igual a 13791,3 $\mathrm{MJ} \mathrm{ha}^{-1}$ (Tabela 10) representa o somatório de DE (Tabela 7), EI (Tabela 8) e EMO (energia empregada na mão-de-obra). Na energia empregada na mão-de-obra

Tabela 10. Energia utilizada (EU), energia extraída (EE), balanço energético (BE) e relação EI / EU do feijoeiro sob lâminas de irrigação (LI)

\begin{tabular}{|c|c|c|c|c|}
\hline \multirow{2}{*}{$\begin{array}{c}\text { 니 } \\
(\mathrm{mm})\end{array}$} & EU & EE & \multirow{2}{*}{ BE } & \multirow{2}{*}{ El / EU } \\
\hline & \multicolumn{2}{|c|}{$\left(\mathrm{MJ} \mathrm{ha}^{-1}\right)$} & & \\
\hline 0 & 13791,3 & 13963,3 & 1,01 & 0,97 \\
\hline 104,1 & 17238,5 & 22212,1 & 1,29 & 0,78 \\
\hline 171,8 & 19480,3 & 27576,5 & 1,42 & 0,69 \\
\hline 192,4 & 20162,5 & 29208,8 & 1,45 & 0,66 \\
\hline 229,2 & 21381,0 & 32124,8 & 1,50 & 0,63 \\
\hline 333,0 & 24818,4 & 40349,9 & 1,63 & 0,54 \\
\hline
\end{tabular}


considerou-se uma necessidade diária de $2000 \mathrm{kcal}(8,38 \mathrm{MJ})$. Como o tempo utilizado na mão-de-obra foi 105,6 ha obteve-se uma EMO equivalente a 110,6 MJ. Nos tratamentos irrigados acrescentaram-se, ao valor de EU igual 13791,3 $\mathrm{MJ}^{-1}$, a EEL e a DE em função das lâminas de irrigação.

$\mathrm{Na}$ Tabela 10, além do balanço energético $(\mathrm{BE}=0,0018 \mathrm{LI}+$ 1,$\left.075 ; \mathrm{R}^{2}=0,9605 ; \mathrm{P}<0,01\right)$, tem-se a relação entre as energias de insumos e utilizada (EI / EU). Pode-se perceber que a relação diminui em função das lâminas de irrigação influenciada pelas energias demandadas por eletricidade e depreciação (Tabela 9) mas não o suficiente para fazer com que os insumos deixem de ter a maior participação na energia utilizada em todos os tratamentos (EI / EU > 0,5).

A irrigação aumentou de 25 a $80 \%$ a entrada de energia (energia utilizada) mas a produtividade (energia extraída) favoreceu o balanço energético $(\mathrm{BE})$ da cultura do feijão irrigado, maior que um (01) em todos os casos, aumentando em função da irrigação (Tabela 8) atingindo 1,63 na aplicação da maior lâmina (333 mm). Comportamento diferente foi verificado por Jordan et al. (2012a), em trabalho conduzido com a cultura do girassol em que o balanço de energia decresceu em função das lâminas de irrigação; ainda para a cultura da mamona, Jordan et al. (2012b) verificaram que o balanço de energia não foi afetado pela irrigação.

\section{CONCLUSÕES}

1. Economicamente, a ausência de irrigação inviabiliza o cultivo do feijoeiro de terceira safra na região Noroeste do Paraná.

2. A irrigação promove viabilidade econômica em curto e longo prazos.

3. A irrigação promove viabilidade energética com acréscimo de balanço de energia em função das lâminas de irrigação.

\section{Literatura Citada}

Albuquerque, F. A.; Beltrão, N. E. de M.; Vale, D. G. Análise energética do algodoeiro na agricultura familiar em diferentes regiões nos estados do Ceará e Mato Grosso do Sul. Campina Grande: EMBRAPA, 2007. 7p. Circular Técnica, n.116.

Albuquerque, P. E. M. Manejo de irrigação. Informe Agropecuário, v.31, p.17-25, 2010.

Arf, O.; Rodrigues, R. A. F.; Sá, M. E.; Buzetti, S.; Nascimento, V. Manejo do solo, água e nitrogênio no cultivo de feijão. Pesquisa Agropecuária Brasileira, v.39, p.131-138, 2004.

Assenheimer, A.; Campos, A. T.; Gonçalves Júnior, A. F. C. Análise energética de sistemas de produção de soja convencional e orgânica. Ambiência, v.5, p.443-455, 2009.

Ávila, M. R.; Barizão, D. A. O.; Gomes, E. P.; Fedri, G.; Albrecht, L. P. Cultivo do feijoeiro no outono/inverno associado à aplicação de bioestimulante e adubo foliar na presença e ausência de irrigação. Scientia Agraria, v.11, p.221-230, 2010.

Campos, A. T.; Campos, A. T. Balanços energéticos agropecuários: Uma importante ferramenta como indicativo de sustentabilidade de agroecossistemas. Ciência Rural, v.34, p.1977-1985, 2004.
Chechetto, R. G.; Siqueira, R.; Gamero, C. A. Balanço energético para a produção de biodiesel pela cultura da mamona. Revista Ciência Agronômica, v.41, p.546-553, 2010.

CONAB - Companhia Nacional de Abastecimento. Acompanhamento da safra brasileira: Nono levantamento, junho, 2010. http://www.conab.gov.br/detalhe. php?c=18596\&t=2 $>20$ Jul. 2010.

COPEL - Companhia Paranaense de Energia. Copel Rural. http://www.copel.com/hpcopel/rural/ > $20 \mathrm{Jul}$. 2010.

EMBRAPA - Empresa Brasileira de Pesquisa Agropecuária. Sistema brasileiro de classificação dos solos. Brasília: EMBRAPA, 2006. 306p.

FINAME - Financiamento de Máquinas e Equipamentos. http://www.caixa.gov.br/pj/pj_comercial/mp/linha_credito/ financiamentos/finame/saiba_mais.asp > 20 Jul. 2010.

Frizzone, J. A.; Andrade Júnior, A. S.; Souza, J. L. M.; Zocoler, J. L. Análise de projetos de irrigação. In: Planejamento de irrigação. Brasília: EMBRAPA, 2005. p.17-58.

Genuchten, M. T. van. A closed equation for predicting the hydraulic conductivity of unsaturated soils. Soil Science Society of America Journal, v.44, p.892-898, 1980.

Gomes, E. P.; Ávila, M. R.; Rickli, M. E.; Petri, F.; Fedri, G. Desenvolvimento e produtividade do girassol sob lâminas de irrigação em semeadura direta na região do Arenito Caiuá, Estado do Paraná. Irriga, v.15, p.373-385, 2010.

Hülsbergen, K. J.; Feil, B.; Biermann, S.; Rathke, G. W.; Kalk, W. D.; Diepenbrock, W. A method of energy balancing in crop production and its application in a long-term fertilizer trial. Agriculture Ecosystems and Environment, v.86, p.303321, 2001.

Jadoski, S. O.; Carlesso, R.; Melo, G. L. Manejo de irrigação para maximização do rendimento de grãos do feijoeiro. Irriga, v.8, p.1-9, 2003.

Johan, J. A.; Silva, M. C. A.; Uribe-Opazo, M. A.; Dalposso, G. H. Variabilidade espacial da rentabilidade, perdas na colheita e produtividade do feijoeiro. Engenharia Agrícola, v.30, p.700-714, 2010.

Jordan, R. A.; Gomes, E. P.; Biscaro, G. A. Impact of irrigation on yield and energy balance of the production of oil and cake of two sunflower varieties. Engenharia Agrícola, v.38, p.1048-1057, 2012a.

Jordan, R. A.; Gomes, E. P.; Biscaro, G. A.; Motomiya, A. V. A.; Geisenhoff, L. Impacto energético da irrigação por gotejamento no cultivo de mamona. Pesquisa Agropecuária Tropical, v.42, p.375-382, 2012 b.

Marques, P. A. A.; Coelho, R. D. Estudo da viabilidade econômica da irrigação da pupunheira para Ilha Solteira SP, Brasil. Ciência Rural, v.33, p.291-297, 2003.

Martin, N. B.; Serra, R.; Antunes, J. F. G.; Oliveira, M. D. M.; Okawa, H. Custos: sistema de custo de produção agrícola. Informações Econômicas, v.24, p.97-122, 1994.

Melo, D.; Pereira, J. O.; Souza, E. G.; Gabriel Filho, A.; Nóbrega, L. H. P.; Pinheiro Neto, R. Energetic balance of soybean and corn production systems in a farm of the west of Paraná, Brazil. Acta Scientiarum Agronomy, v.29, p.173-178, 2007.

R. Bras. Eng. Agríc. Ambiental, v.17, n.8, p.835-842, 2013. 
Rapassi, R. M. A.; Sá, M. E.; Tarsitano, M. A. A.; Carvalho, M. A. C.; Proença, E. R.; Neves, C. M. T. C.; Colombo, E. C. M. Análise econômica comparativa após um ano de cultivo do feijoeiro irrigado, no inverno, em sistemas de plantio convencional e direto, com diferentes fontes e doses de nitrogênio. Bragantia, v.62, p.397-404, 2003.

Rios, A. O.; Abreu, C. M. P.; Corrêa, A. D. Efeito de estocagem e das condições de colheita sobre algumas propriedades físicas, químicas e nutricionais de três cultivares de feijão. Ciência e Tecnologia de Alimentos, v.23, p.39-45, 2003.

Santana, M. J.; Carvalho, J. A.; Andrade, M. J. B.; Gervásio, G., G., Braga, J. C.; Lepri, E. B. Viabilidade técnica e econômica da aplicação de água na cultura do feijoeiro comum. Ciência \& Agrotecnologia, v.33, p.532-538, 2009a.
Santana, M. J.; Silveira, A. L.; Camargos, C. R.; Braga, J. C. Tensão de água no solo e doses de nitrogênio para a cultura do feijoeiro comum. Irriga, v.14, p.518-532, 2009 b.

Saturnino, H. M.; Christofidis, D.; Costa, E. L.; Reis, J. B. S. Agricultura irrigada: Oportunidades e desafios. Informe Agropecuário, v.31, p.101-109, 2010.

SEAB - Secretaria da Agricultura e do Abastecimento do Paraná. Produção Agrícola Paranaense por Município últimas cinco safras. http://www.seab.pr.gov.br/modules/ conteudo/conteudo $>20$ Jul. 2010.

Silva, F. de A. S.; Azevedo, C. A. V. de. Assistat 7.6 beta: Assistência estatística. < http: www.assistat.com>20 Jul. 2010.

Silveira, P. M.; Silva, O. F.; Stone, L. F.; Silva, J. G. Efeitos do preparo do solo, plantio direto e de rotações de culturas sobre o rendimento e a economicidade do feijoeiro irrigado. Pesquisa Agropecuária Brasileira, v.36, p.257-263, 2001. 